\title{
NON-IDEAL SAMPLING AND ADAPTED RECONSTRUCTION USING THE STOCHASTIC MATÉRN MODEL
}

\author{
Sathish Ramani, Dimitri Van De Ville and Michael Unser \\ Biomedical Imaging Group, \\ Ecole Polytechnique Fédérale de Lausanne (EPFL), \\ CH-1015 Lausanne, Switzerland.
}

\begin{abstract}
The Matérn class is a parametric family of autocorrelation functions that is commonly used in geostatistics. We argue that a generalized, anisotropic version of this model is suitable for capturing the correlation structure of a variety of natural images. We specify the optimal space for the MMSE reconstruction of stochastic Matérn signals from their uniformlysampled noisy measurements (generalized sampling problem). We prove that the optimal reconstruction space is generated by the multi-integer shifts of a Matern function which form a Riesz basis. Based on this representation, we propose a practical filter-based reconstruction method that relies on the prior identification of the Matérn parameters from the measured data. We present experimental results to justify the use of the Matérn model and to demonstrate the performance of our signal-adapted reconstruction technique.
\end{abstract}

\section{INTRODUCTION}

The Matérn class is a parametric family of autocorrelation functions that is of practical value in geostatistics for the prediction/estimation — also referred to as kriging — of spatial data [1]. To the best of our knowledge, this signal model has not yet received much attention in the signal processing literature. Recently, an extensive overview of kriging was presented in [2], but it did primarily focus on the exponential case-the simplest instance of the Matérn model [1]. The Matérn class can also be viewed as a (fractional) generalization of the Markov Random Field model which is often used for modeling images [3]. Also relevant is the observation that many natural images exhibit a $1 /\|\boldsymbol{\omega}\|^{\tau}$-like spectral decay [4], which is compatible with the Matérn model.

Our purpose in this paper is to present further arguments in favor of the Matérn class and to demonstrate its usefulness for stochastic image modeling and processing. We will concentrate on the 2D case and consider the generalized sampling problem that is summarized in Fig. 1. Specifically, we assume that the signal $s(\mathbf{x})$ that we are sampling is a realization of a Matérn stochastic process. The measurements are affected by blur (prefilter $h$ ) and additive noise $n$. Our goal is to obtain the best signal reconstruction $s_{\text {rec }}(\mathbf{x})$ that minimizes the mean square estimation error $E\left\{\left|s_{\text {rec }}(\mathbf{x})-s(\mathbf{x})\right|^{2}\right\}$ for all $\mathbf{x} \in \mathbb{R}^{d}$. In our previous work, we have shown that this could be achieved by means of a hybrid Wiener filter that accepts discrete input data $g[\mathbf{k}]$ and determines the op-

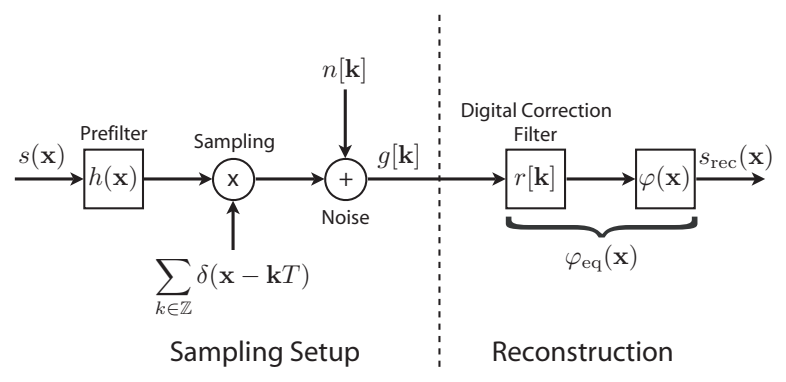

Fig. 1. Practical Sampling Setup and Reconstruction.

timal signal coefficients in a reconstruction space spanned by the multi-integer shifts of a suitable generating function $\varphi(\mathbf{x})$ that is generally not bandlimited $[5,6]$. Our main contribution here will be to introduce an extended version of the Matérn power spectral density (PSD) that can account for image anisotropies, and to rigorously specify a Riesz basis (via its generating function $\varphi(\mathbf{x})$ ) for the corresponding optimal reconstruction space. We will demonstrate the suitability of this model for natural images. To make the method practical, we also introduce an estimation procedure for identifying the Matérn parameters and the noise variance from the noisy measurements.

In the following section, we briefly discuss the sampling problem for stationary stochastic signals, its optimal solution, and the mathematical specification of a stable reconstruction basis. In Section 3, we present the Matérn PSD and its anisotropic extension and prove that the underlying correlation function generates a Riesz basis of the optimal reconstruction space. In Section 4, we demonstrate the aptness of the model in capturing the correlation structure of images and how it can be used for improved image reconstruction. We give a brief description of our parameter-estimation procedure and present experimental results that justify the use of the Matérn model for reconstruction. We display examples where our blind estimation method is competitive with the best possible bandlimited reconstruction (oracle solution).

\section{SAMPLING AND RECONSTRUCTION}

Our measurement model follows the generalized sampling setup in Fig. 1,

$$
g[\mathbf{k}]=(h \star s)(\mathbf{k})+n[\mathbf{k}],
$$


where $s(\mathbf{x})$ is the continuous-space signal that is a realization of a zero-mean stationary process with autocorrelation $c_{s s}(\mathbf{x}),(h \star s)(\mathbf{k})$ are the samples of prefiltered signal, and $n[\mathbf{k}]$ is a discrete additive, zero-mean white noise component with variance $\sigma^{2}$. The reconstruction space,

$$
V(\varphi)=\left\{f(\mathbf{x})=\sum_{\mathbf{k} \in \mathbb{Z}^{d}} c[\mathbf{k}] \varphi(\mathbf{x}-\mathbf{k}) \mid c \in \ell_{2}\left(\mathbb{Z}^{d}\right)\right\},
$$

is spanned by the multi-integer shifts of the generating function $\varphi(\mathbf{x})$. For this representation to be unambiguous, we re-

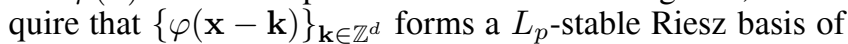
$V(\varphi)$, for $1 \leq p \leq \infty$. This is ensured provided that the two admissibility conditions are met:

$$
\begin{aligned}
& 0<A_{\varphi}^{2} \leq \sum_{\mathbf{k} \in \mathbb{Z}^{d}}|\hat{\varphi}(\boldsymbol{\omega}+2 \pi \mathbf{k})|^{2} \leq B_{\varphi}^{2}<\infty
\end{aligned}
$$

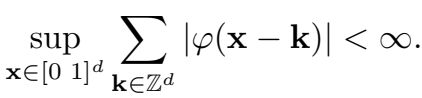

where $\hat{\varphi}(\boldsymbol{\omega})$ is the Fourier transform of $\varphi$ [7].

The next theorem states that the MMSE solution to the reconstruction problem lies in an integer shift-invariant reconstruction space of the type defined above. It also gives closed-form expressions for the optimal generator $\varphi(\mathbf{x})$ and the optimal digital correction filter $r[\mathbf{k}]$ in Fig. 1.

Theorem 1 [5] Given the measurement model(1), the MMSE estimate of $s(\mathbf{x})$ lies in the integer-shift-invariant space generated by

$$
\varphi(\mathbf{x})=\left(\bar{h} \star c_{s s}\right)(\mathbf{x})
$$

where $\bar{h}(\mathbf{x})=h(-\mathbf{x})$. The optimal solution is

$$
s_{\mathrm{rec}}(\mathbf{x})=\sum_{\mathbf{k} \in \mathbb{Z}^{d}}(r * g)[\mathbf{k}] \varphi(\mathbf{x}-\mathbf{k}),
$$

where the Fourier transform of the digital correction filter $r[\mathbf{k}]$ is

$$
R\left(e^{j \boldsymbol{\omega}}\right)=\frac{1}{\sum_{\mathbf{k} \in \mathbb{Z}^{d}} \hat{\varphi}(\boldsymbol{\omega}+2 \pi \mathbf{k}) \hat{h}(\boldsymbol{\omega}+2 \pi \mathbf{k})+\sigma^{2}} .
$$

\section{MATÉRN CLASS OF PSD}

In this section, we present the isotropic and anisotropic (more general) Matérn power spectral densities and show that the Matérn correlations are valid (i.e., $L_{p}$-stable) generators of the reconstruction space.

\subsection{Isotropic Matérn PSD}

The multidimensional Matérn PSD is defined as (cf. $[1,8]$ ),

$$
\begin{aligned}
& \hat{\phi}_{\text {iso }}(\boldsymbol{\omega})=\frac{\sigma_{0}^{2}}{\left(\alpha^{2}+\|\boldsymbol{\omega}\|^{2}\right)^{\nu+d / 2}}, \\
& \sigma_{0}^{2}>0, \text { is the innovation variance, } \\
& \alpha>0, \text { represents the autocovariance range, } \\
& \nu>0, \text { measures the smoothness of the process. }
\end{aligned}
$$

It is an isotropic function, meaning that the process does not possess any preferred "direction" of evolution. The corresponding autocorrelation function of the process is given by,

$$
\phi_{\text {iso }}(\mathbf{x})=\frac{\sigma_{0}^{2} \sqrt{\pi^{d}}}{2^{\nu-1} \Gamma(\nu+d / 2) \alpha^{2 \nu}}(\alpha\|\mathbf{x}\|)^{\nu} \mathcal{K}_{\nu}(\alpha\|\mathbf{x}\|),
$$

where $\mathcal{K}_{\nu}$ is the modified Bessel function of the second kind. It is known that $\mathcal{K}_{\nu}(u)>0$ for positive $u$, and that it rapidly decays for increasing $u$. We now prove that $\phi_{\text {iso }}(\mathbf{x})$ and its integer-shifts form a $L_{p}$-stable Riesz basis by checking for the admissibility conditions (3) and (4).

Proposition 1 For all $\nu>\frac{-d+1}{4}$, the function $\phi_{\text {iso }}(\mathbf{x})$ has Riesz bounds given by

$$
\begin{aligned}
& 0<A_{\phi_{\text {iso }}}^{2} \leq \sum_{\mathbf{k} \in \mathbb{Z}^{d}}\left|\hat{\phi}_{\text {iso }}(\boldsymbol{\omega}+2 \pi \mathbf{k})\right|^{2} \leq B_{\phi_{\text {iso }}}^{2}<+\infty \\
& A_{\phi_{\text {iso }}}^{2} \geq \frac{\sigma_{0}^{4}}{\left(\alpha^{2}+4 \pi^{2} d\right)^{2 \nu+d}} \\
& B_{\phi_{\text {iso }}}^{2} \leq\left(\frac{\sigma_{0}^{4}}{\alpha^{4 \nu+2 d}}+\frac{\sigma_{0}^{4} 2^{d} \pi^{d / 2} \zeta\left(2 \nu+\frac{d+1}{2}\right)}{2 \Gamma\left(\frac{d}{2}+1\right)(2 \pi)^{4 \nu+2 d}}\right)
\end{aligned}
$$

where $\zeta(\cdot)$ is the Riemann-zeta function. For $\nu>0$, the function $\phi_{\text {iso }}(\mathbf{x})$ satisfies the $L_{p}$-stability condition, that is,

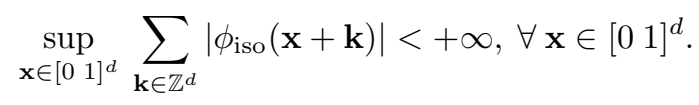

Note that the above results hold for any number of dimensions, $d \geq 1$.

\subsection{Anisotropic Matérn PSD}

We introduce an anisotropic version of the Matérn PSD by applying a similarity transform (scaling and rotation) of the coordinate system. The 2-D version of the model is

$$
\begin{gathered}
\hat{\phi}_{\text {ani }}(\boldsymbol{\omega})=\frac{\sigma_{0}^{2}}{(\alpha^{2}+\underbrace{\mathcal{A} \cdot \omega_{1}^{2}+\mathcal{B} \cdot \omega_{2}^{2}+\mathcal{C} \cdot \omega_{1} \omega_{2}}_{\omega_{e}^{2}(\boldsymbol{\omega})})^{\nu+1}}, \\
\mathcal{A}=\left(\cos ^{2}(\theta)+\frac{\sin ^{2}(\theta)}{(1-\eta)^{2}}\right), \mathcal{B}=\left(\frac{\cos ^{2}(\theta)}{(1-\eta)^{2}}+\sin ^{2}(\theta)\right), \\
\mathcal{C}=-\sin (\theta) \cos (\theta)\left(\frac{1}{(1-\eta)^{2}}-1\right),
\end{gathered}
$$

where $\theta$ is the angle between the principal direction of anisotropy and the abscissa, and $0 \leq \eta<1$ is the ellipticity of the anisotropy. When $\eta=0$, there is no anisotropy and (12) reduces to (8) for $d=2$, irrespective of the value of $\theta$. Examples of isotropic and anisotropic Matérn PSDs are shown in Figs. 2 and 3 (parameters: $\sigma_{0}=1, \alpha=0.25, \nu=0.5$ ). The generalized Matérn function, $\phi_{\text {ani }}(\mathbf{x})$ also generates an $L_{p}$-stable Riesz basis. 


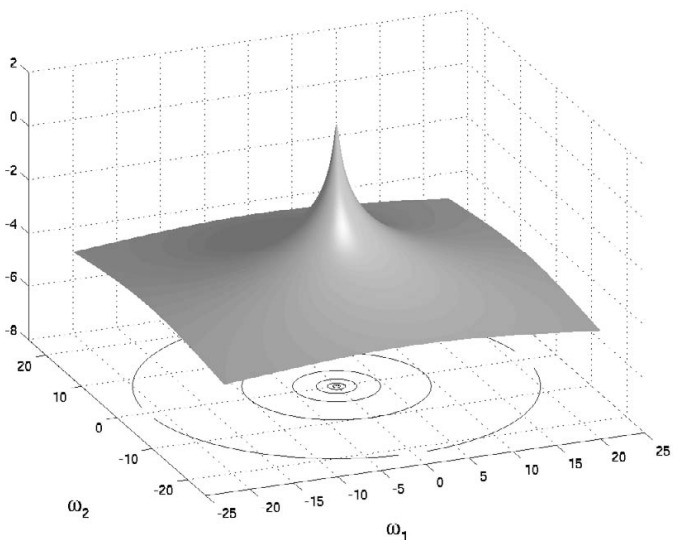

Fig. 2. Log plot of Isotropic Matérn PSD in 2-D.

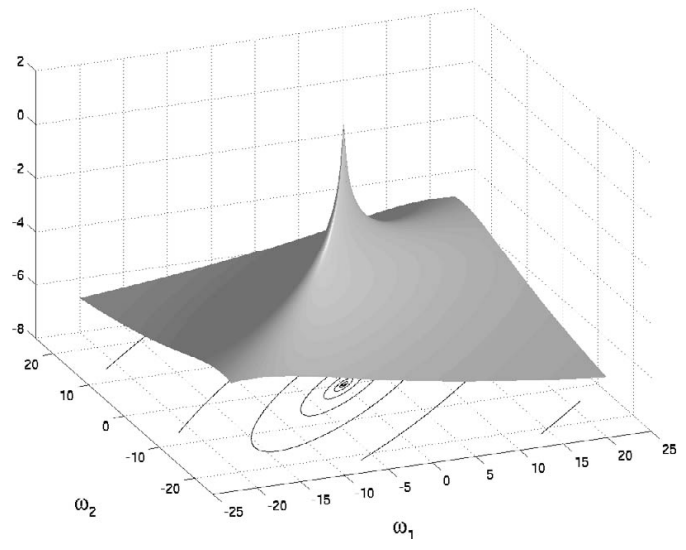

Fig. 3. Log plot of Anisotropic Matérn PSD in 2-D.

Proposition 2 For all $\nu>-\frac{1}{4}$, the function $\phi_{\text {ani }}(\mathbf{x})$ has Riesz bounds given by

$$
\begin{aligned}
& 0<A_{\phi_{\text {ani }}}^{2} \leq \sum_{\mathbf{k} \in \mathbb{Z}^{2}}\left|\hat{\phi}_{\mathrm{ani}}(\boldsymbol{\omega}+2 \pi \mathbf{k})\right|^{2} \leq B_{\phi_{\mathrm{ani}}}^{2}<+\infty \\
& A_{\phi_{\mathrm{ani}}}^{2} \geq \frac{\sigma_{0}^{4}}{\left(\alpha^{2}+8 \pi^{2} b^{2}\right)^{2 \nu+2}} \\
& B_{\phi_{\mathrm{ani}}}^{2} \leq\left(\frac{\sigma_{0}^{4}}{\alpha^{4 \nu+4}}+\frac{\sigma_{0}^{4} 2 \pi \zeta\left(2 \nu+\frac{3}{2}\right)}{(2 a \pi)^{4 \nu+4}}\right)
\end{aligned}
$$

for some $a, b>0$. For $\nu>0, \phi_{\text {ani }}(\mathbf{x})$ satisfies the $L_{p^{-}}$ stability condition, that is,

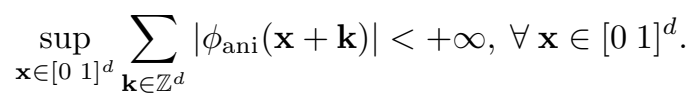

Since, $\varphi(\mathbf{x})=\left(\bar{h} \star c_{s s}\right)(\mathbf{x})$ in (5) is the generating function of the optimal reconstruction space, we would like it to be admissible for $c_{s s}(\mathbf{x})=\phi_{\mathrm{ani}}(\mathbf{x})$.

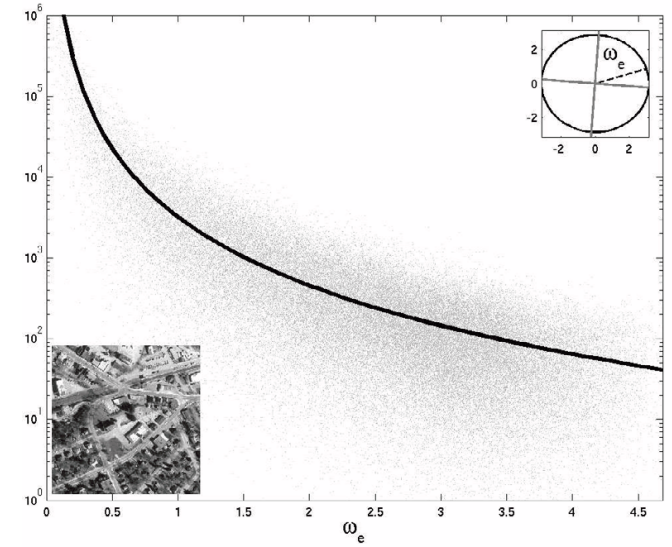

Fig. 4. Aerial View image: Isotropic PSD, $\eta=0.098, \theta=$ $176.765^{\circ}, \sigma_{0}=56.604, \alpha=0.021, \nu=0.410$.

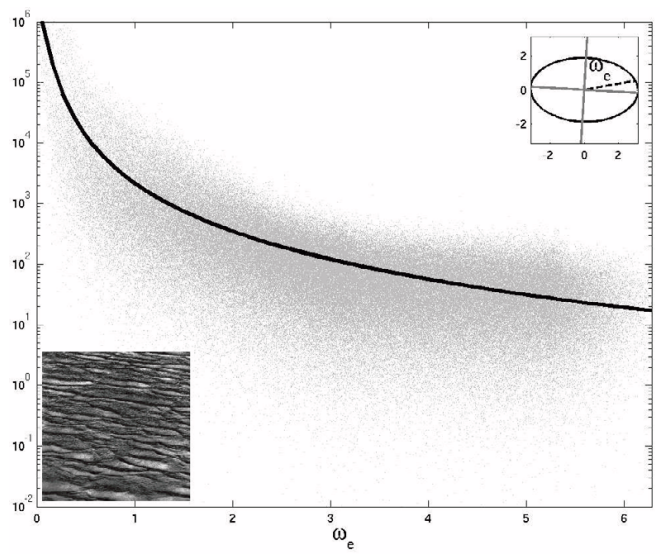

Fig. 5. D37 Brodatz texture: Anisotropic PSD, $\eta=0.407$, $\theta=176.674^{\circ}, \sigma_{0}=46.811, \alpha=0.083, \nu=0.320$.

Proposition 3 If $h(\mathbf{x}) \in L_{1}\left(\mathbb{R}^{d}\right)$ and the set $A=\left\{\mathbf{k} \in \mathbb{Z}^{d}\right.$ : $\left.\hat{h}(\boldsymbol{\omega}+2 \pi \mathbf{k}) \neq 0 \forall \boldsymbol{\omega} \in[02 \pi)^{d}\right\} \neq \emptyset$, then $\varphi(\mathbf{x})=(\bar{h} \star$ $\left.\phi_{\mathrm{ani}}\right)(\mathbf{x})$, is also an admissible generating function; i.e., $\varphi(\mathbf{x})$ generates an $L_{p}$-stable Riesz basis.

\section{RECONSTRUCTION USING MATÉRN MODEL}

We now propose to use the generating function $\varphi(\mathbf{x})=(\bar{h} \star$ $\left.\phi_{\text {ani }}\right)(\mathbf{x})$ to specify the optimal reconstruction space. We consider a texture (Brodatz-D37) and a natural image (Aerial View) for this purpose. The gray dots in Figs. 4 and 5 indicate the values of the periodogram of the images as a function of $\omega_{e}$, which is the distance from the origin to a point whose locus is an ellipse (see insets). The solid line in each figure is the generalized Matérn model as a function of $\omega_{e}$ fit to the periodogram of the image. It is clearly seen that the generalized Matérn model captures the trend of the periodograms while accounting for the anisotropy in D37 texture via the ellipticity parameter $\eta$. 


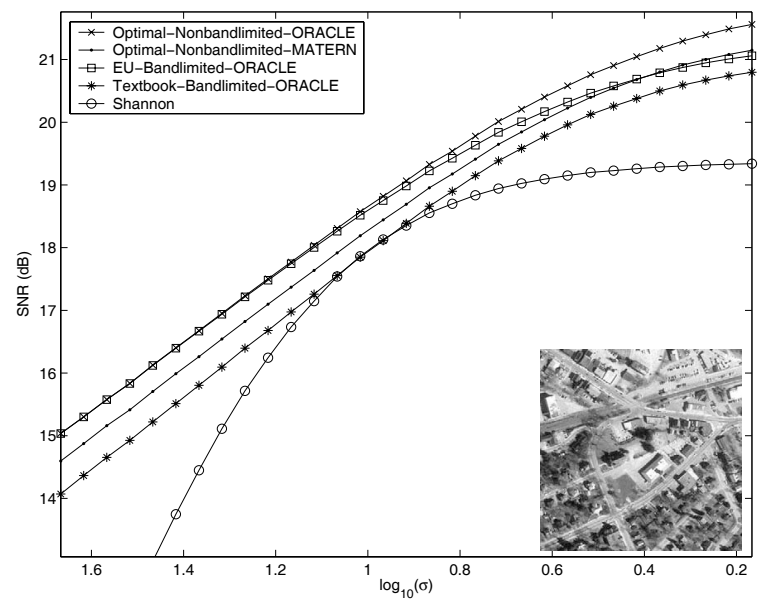

Fig. 6. Performance of reconstruction methods: SNR Variation for Aerial view.

\subsection{Estimation of Matérn parameters}

We estimate the parameters in $\phi_{\text {ani }}$ viz-a-viz, ellipticity $\eta$, angle $\theta$ and the Matern parameters $\sigma_{0}, \alpha, \nu$ and also the noise variance $\sigma^{2}$ by performing a non-linear least squares minimization of the following cost function,

$\epsilon=\sum_{\boldsymbol{\omega} \in(0,2 \pi)^{2}}\left[\log \left(\tilde{\Phi}(\boldsymbol{\omega})+\tilde{\sigma}^{2}\right)-\log \left(\hat{\Phi}_{n p}(\boldsymbol{\omega})\right)-\gamma_{e}\right]^{2}$,

where $\tilde{\Phi}(\boldsymbol{\omega})=\sum_{\mathbf{k} \in \mathbb{Z}^{d}}|\hat{h}(\boldsymbol{\omega}+2 \pi \mathbf{k})|^{2} \hat{\phi}_{\text {ani }}(\boldsymbol{\omega}+2 \pi \mathbf{k}), \hat{\Phi}_{n p}$ is the periodogram of the noisy measurements $g[\mathbf{k}]$ and $\gamma_{e}=$ 0.57721 , is the Euler-Mascheroni constant, to account for the bias due the log function. We exclude $\omega=0$ in the summation to avoid spurious entries for the DC component in the periodogram $\hat{\Phi}_{n p}$.

\subsection{Results}

In our experiments, the reference scale for the signal and its reconstruction was $T=1$. The signal was blurred with a standardized Gaussian prefilter and downsampled by a factor of two. The measurements were corrupted by the addition of various levels of white Gaussian noise.

The noise variance and the parameters of the anisotropic Matérn PSD were estimated from these noisy measurements. The reconstruction of the images at original resolution was performed according to the procedure outlined in Theorem 1 using $c_{s s}=\phi_{\text {ani }}$ with the estimated parameters. This was compared with the optimally-compensated bandlimited reconstruction, namely EU-bandlimited [6], the textbook implementation of the Wiener filter associated with a bandlimited version of the PSD, and Sinc-interpolation without compensation (Shannon's method). Some of these are referred to as ORACLE-solutions since they used the noise-free PSD to derive the best possible reconstruction filter. Figs. 5 and 6 show the SNR of reconstruction for various noise levels. We see that we perform consistently better than the Oracle Textbookbandlimited and Sinc-interpolation methods. We even outperform the Oracle EU-bandlimited method at lower noise levels.

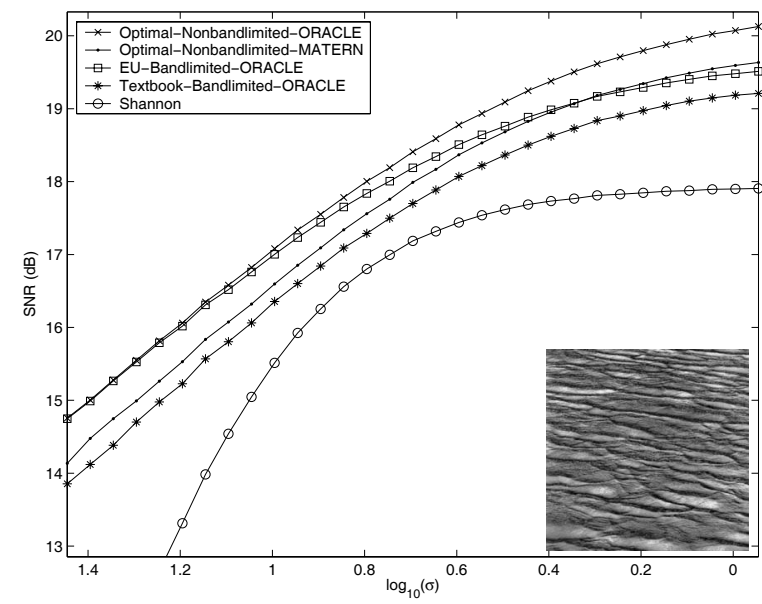

Fig. 7. Performance of reconstruction methods: SNR Variation for D37 Brodatz texture.

\section{CONCLUSIONS}

In this work, we have suggested a practically viable method for the MMSE reconstruction of images from their prefiltered noisy samples using the Matérn stochastic model. We have proposed the use of an anisotropic Matérn function which is a more general model that accounts for anisotropies in images. We also give illustrations to justify the use of the anisotropic Matérn PSD and demonstrate the superiority of our non-bandlimited optimal reconstruction using the anisotropic Matérn function in comparison to the oracle-bandlimited reconstructions.

\section{ACKNOWLEDGEMENTS}

This work is funded in part by the grant 200020-109415 from the Swiss National Science Foundation (SNSF).

\section{REFERENCES}

[1] M. L. Stein, Interpolation of Spatial Data-Some Theory for Kriging, Chapter 2, Springer-Verlag, New York, 1999.

[2] J. R. Alzola, C. A. López and C. F. Westin, "Kriging filters for multidimensional signal processing," Signal Processing, 85, 2004, pp. 413 439.

[3] A. J. Gray, J. W. Kay, D. M. Titterington, "An empirical study of the simulation of various models used for images," IEEE Trans. Pattern Anal. Mach. Intell., vol.16, no. 5, May 1994, pp. 507 - 513.

[4] B. Pesquet-Popescu and J. Lévy Véhel, "Stochastic fractal models for image processing," IEEE Signal Process. Mag., vol. 19, no. 5, pp. 4862, 2002.

[5] S. Ramani, D. Van De Ville, M. Unser, "Sampling in Practice: Is the Best Reconstruction Space Bandlimited?,' ICIP'05, Genova, Italy, September 11-14, 2005, pp. II-153 - II-156.

[6] Y. C. Eldar and M. Unser, "Non-ideal sampling and Interpolation from noisy observations in Shift-Invariant spaces," IEEE Trans. Signal Process., in press.

[7] A. Aldroubi and K. Grochenig, "Nonuniform sampling and reconstruction in shift invariant spaces," SIAM Review, vol. 43, pp. 585-620, 2001.

[8] B. Whitcher, "Wavelet-based Bootstrapping of Spatial Patterns on a Finite Lattice,'Computational Statistics \& Data Analysis, in press. 